\title{
PHF8 is a histone H3K9me2 demethylase regulating rRNA synthesis
}

Ziqi Zhu, Yanru Wang, Xia Li, Yiqin Wang, Longyong Xu, Xiang Wang, Tianliang Sun, Xiaobin Dong, Lulu Chen, Hailei Mao, Yi Yu, Jinsong Li, Pin Adele Chen, Charlie Degui Chen

Cell Research (2010) 20:970. doi:10.1038/cr.2010.112; published online 2 August 2010

Correction to: (2010) 20:794-801. doi:10.1038/cr.2010.75; published online 8 June 2010

The authors report that the name of the third author from the last was incorrect in the article. The correct name is Jinsong Li, not Jingsong Li. The authors apologize for the error. 